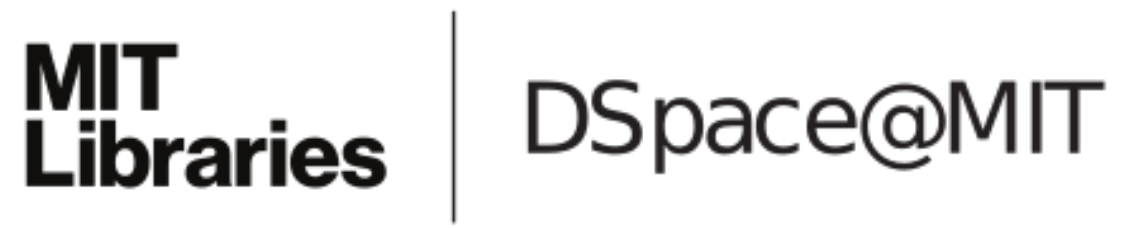

\author{
MIT Open Access Articles
}

Solving the Trivial Crossing Problem While Preserving the Nodal Symmetry of the Wave Function

The MIT Faculty has made this article openly available. Please share how this access benefits you. Your story matters.

Citation: Lee, Elizabeth M. Y. Lee and Willard, Adam P. "Solving the Trivial Crossing Problem While Preserving the Nodal Symmetry of the Wave Function." Journal of Chemical Theory and Computation 15, 8 (July 2019): 4332-4343 (c) 2019 American Chemical Society.

As Published: http://dx.doi.org/10.1021/acs.jctc.9b00302

Publisher: American Chemical Society (ACS)

Persistent URL: https://hdl.handle.net/1721.1/124405

Version: Final published version: final published article, as it appeared in a journal, conference proceedings, or other formally published context

Terms of use: Creative Commons Attribution-NonCommercial-NoDerivs License 


\title{
Solving the Trivial Crossing Problem While Preserving the Nodal Symmetry of the Wave Function
}

\author{
Elizabeth M. Y. Lee ${ }^{\dagger}$ and Adam P. Willard**;(0) \\ ${ }^{\dagger}$ Pritzker School of Molecular Engineering, The University of Chicago, Chicago, Illinois 60637, United States \\ ${ }^{\ddagger}$ Department of Chemistry, Massachusetts Institute of Technology, Cambridge, Massachusetts 02139, United States
}

\section{Supporting Information}

ABSTRACT: In an adiabatic mixed quantum-classical simulation, the avoided crossing of weakly coupled eigenstates can lead to unphysical discontinuities in wave function dynamics, otherwise known as the trivial crossing problem. A standard solution to the trivial crossing problem eliminates spatial discontinuities in wave function dynamics by imposing changes to the eigenstate of the wave function. In this paper, we show that this solution has the side effect of introducing transient discontinuities in the nodal symmetry of the wave
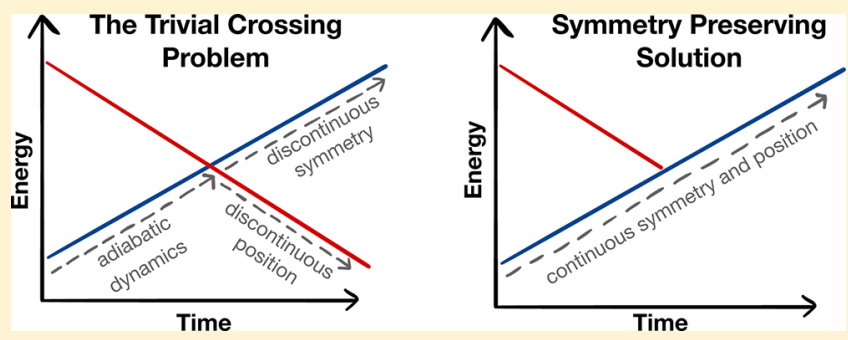
function. We present an alternative solution to the trivial crossing problem that preserves both the spatial and nodal structure of the adiabatic wave function. By considering a model of exciton dynamics on conjugated polymer systems, we show that failure to preserve wave function symmetry yields exciton dynamics that depends unphysically on polymer system size. We demonstrate that our symmetry-preserving solution to the trivial crossing problem yields more realistic dynamics and can thus improve the accuracy of simulations of larger systems that are prone to the trivial crossing problem.

\section{INTRODUCTION}

Many approaches to mixed quantum-classical simulation are based on the use of adiabatic dynamics, where the classical degrees of freedom (e.g., the nuclei) evolve according to the potential energy surface of a single specific eigenstate of the quantum subsystem. If the quantum subsystem has multiple eigenstates, then the dynamics of the classical subsystem can lead to variations in eigenstate energies and the emergence of avoided crossings, as illustrated in Figure 1a. As a system moves along a single eigenstate through a typical avoided crossing, the adiabatic wave function transforms gradually from one eigenstate to the other in a manner that can be expressed as a linear combination of the two corresponding diabatic states, as shown in Figure 1c. However, in the limit that the diabatic states are uncoupled, the quantum subsystem will exhibit a near-discontinuous transition at the crossing point, where the eigenstates are essentially degenerate in energy, as depicted in Figure 1b. This abrupt unphysical change in the characteristics of the adiabatic wave function due to the crossing of eigenstates, as illustrated in Figure 1d, is known as the trivial crossing problem (TCP). ${ }^{1-9}$

A common solution to the TCP is to force the quantum subsystem to change eigenstates at the crossing point in order to maintain the continuity of the adiabatic wave function. ${ }^{2,10,11}$ However, this type of approach necessarily introduces a discontinuous transition in the number of nodes in the adiabatic wave function. In systems that require long time dynamics or that feature multiple trivial crossings, we show that this change in nodal symmetry can lead to inaccurate and inconsistent electronic adiabatic dynamics. In this Article, we present a solution to the TCP that preserves the symmetry of the adiabatic wave function in simulations of adiabatic dynamics. We demonstrate our approach and highlight its utility by applying it to model the dynamics of electronically excited conjugated polymer systems.

The TCP represents a breakdown in the adiabatic approximation. In the adiabatic limit, where classical degrees of freedom evolve infinitely slowly, the wave function of the quantum subsystem can instantaneously adjust to the motion of classical degrees of freedom and can thus access arbitrarily large changes in electronic state through arbitrarily small electronic couplings. Away from this limit, however, nonadiabatic effects are required to accurately represent the state of the system as it travels through and exits a trivial crossing. Nonadiabatic effects enable a wave function to transform in response to variations in the electronic energy levels and couplings by spreading across different eigenstates of the quantum subsystem. ${ }^{12}$ In the case of a trivial crossing, this transformation could prevent the occurrence of unphysical discontinuities and effectively preserves the diabatic characteristics of the wave function. $5,7,9$

In simulations of adiabatic dynamics (i.e., where the wave function of the quantum subsystem is at all times represented by a single eigenstate), it has been proposed that these nonadiabatic effects can be effectively captured by imposing an

Received: March 25, 2019

Published: July 15, 2019 


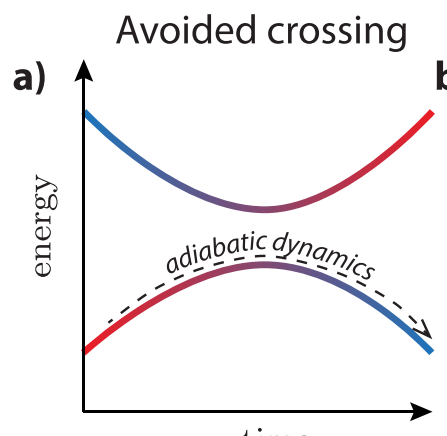

time
Trivial crossing

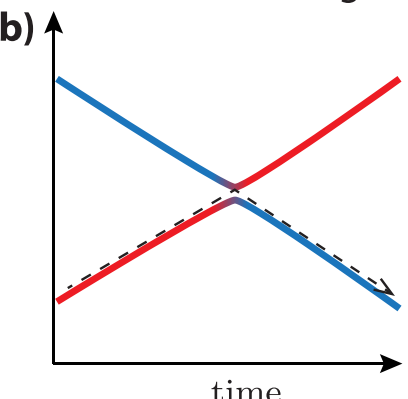

time
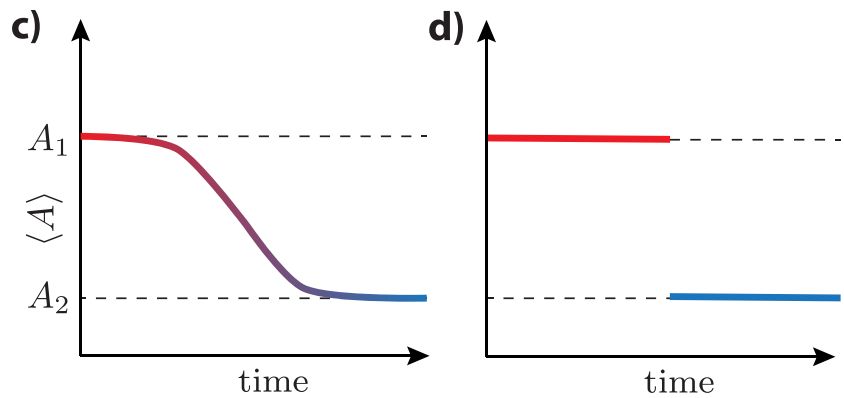

Figure 1. Panels a and $b$ illustrate the energy levels for two eigenstates of a quantum subsystem moving through a traditional avoided and weakly coupled trivial crossing, respectively. Dashed black arrows indicate the path following a simulation of adiabatic dynamics on the lowest energy eigenstate of the quantum subsystem. Panels $c$ and $d$ depict how the physical character of the quantum subsystem, as represented by an observable quantity $\langle A\rangle$, changes for the systems represented by panels a and $\mathrm{b}$, respectively. $A_{1}$ and $A_{2}$ indicate the values of $A$ in two diabatic states. The line color in each panel represents the diabatic identity of each eigenstate or observable.

appropriate change of eigenstate for the wave function as it passes through a trivial crossing. This approach to preventing the trivial crossing problem has been widely adopted because it provides an efficient solution without the need to perform computationally expensive nonadiabatic dynamics. Methods based on this approach typically involve two steps. First, a trivial crossing is identified on the basis of the occurrence of an abrupt change in the adiabatic wave function from one time step to the next. Second, a new eigenstate is chosen such that this change is effectively minimized. Changes in wave function character are quantified in a variety of ways, for example, by computing the overlap integral of eigenstates at sequential points in time. ${ }^{2,10,11,13}$ Variations of this approach include the consideration of additional factors such as energy gaps between two eigenstates, ${ }^{1}$ nonadiabatic coupling vectors, ${ }^{1,4,10,14}$ or differences in calculated surface hopping probabilities. ${ }^{6}$ Other methods circumvent the trivial crossing problem by identifying problematic eigenstates and artificially forbidding their occupation. ${ }^{13-15}$ Notably, in these methods, the forbidden states still belong to the quantum subsystem, so preventing their occupation can still require the reassignment of the wave function eigenstate.

Changing the eigenstate of a wave function necessarily results in a change of the number of nodes in that wave function, because the nodal structure of a given eigenstate depends on its eigenstate index, with the first (i.e., lowest energy) eigenstate having zero nodes, the second having one node, and so on. ${ }^{16}$ In simulations of adiabatic dynamics, a change in nodal structure can lead to impulsive and physically unwarranted changes in the interactions between the classical and quantum subsystems.

In this Article, we present a solution to the trivial crossing problem that eliminates the need to change the eigenstate index of the adiabatic wave function through dynamically inert modifications of the Hamiltonian of the quantum subsystem. Section II introduces the general theoretical framework for this method. Sections III and IV present the application of this method in simulating exciton dynamics on extended conjugated polymer chains. Section V highlights the importance of preserving the nodal symmetry of wave function. Finally, we summarize and discuss implications of our method for large-scale adiabatic simulations.

\section{MODIFYING THE HAMILTONIAN TO ELIMINATE TRIVIAL CROSSINGS}

II.A. General Theoretical Framework for Two-State Systems. Our solution to the trivial crossing problem is to modify the system's Hamiltonian so that purely adiabatic dynamics is free from the occurrence of trivial crossings. In this section, we present a general theoretical formalism for constructing such a modified Hamiltonian. We begin by considering a system comprised of a quantum subsystem and a classical subsystem that are coupled and subject to the BornOppenheimer approximation. That is, we assume that the quantum subsystem is defined by the Hamiltonian $\hat{H}[\mathbf{x}(t)]$, where $\mathbf{x}(t)$ denotes the time dependent configuration of the classical subsystem. An adiabatic state of the quantum subsystem, denoted by $\left|\Psi_{i}\right\rangle$, and its associated energy level, denoted by $\lambda_{i}$, are given by the solution to the timeindependent Schrödinger equation

$$
\hat{H}\left|\Psi_{i}\right\rangle=\lambda_{i}\left|\Psi_{i}\right\rangle
$$

for $i=1,2, \ldots$. Adiabatic states are generally indexed in order of increasing $\lambda$, i.e., with $\left|\Psi_{1}\right\rangle$ representing the eigenstate with the lowest eigenvalue.

We assume that the classical subsystem evolves under the combined influence of a potential energy surface, such as a molecular mechanics force field, which only depends on $\mathbf{x}(t)$, and the forces from the quantum subsystem, which are given by the Hellmann-Feynman formula

$$
F_{\alpha}^{(n)}=-\left\langle\Psi_{k}\left|\frac{\partial \hat{H}}{\partial x_{\alpha}}\right| \Psi_{k}\right\rangle
$$

where $x_{\alpha}$ denotes the position of the $\alpha$ th classical degree of freedom. Notably, this expression describes the specific contribution of the $n$th adiabatic state to the overall nuclear forces. For example, the molecular mechanics force field approximates the electronic ground state forces and the Hellmann-Feynman forces approximate the difference in the ground and excited state forces. The formalism we present below is designed specifically for systems that are initialized with the quantum subsystem in the $n=1$ state; however, the methodology can be easily generalized to handle arbitrary (i.e., $n \neq 1)$ initial conditions. We treat only two-state crossings, noting that in many cases a multistate crossing can be reduced to a series of two-state crossings by decreasing the propagation time step.

Like other proposed solutions to the trivial crossing problem, our method involves two steps. First, we detect the occurrence of a trivial crossing. Second, we correct the dynamics to eliminate its effect. Detecting the crossing of two 
states, where the adiabatic wave function is rapidly transforming in time, is straightforward; however, distinguishing a trivial crossing from a physical one (e.g., differentiating Figure $1 \mathrm{a}$ from Figure $1 \mathrm{~b}$ ) can be challenging because the definition of a trivial crossing is inherently ambiguous. ${ }^{17}$ To address this challenge, we use a diabatization approach to quantify the coupling between crossing states, and we use this coupling as a basis to differentiate trivial from avoided crossings.

To illustrate the concept behind our approach, consider a mixed quantum-classical system with a two-state quantum subsystem that is represented by two time independent basis states (i.e., diabats), $\left|\phi_{a}\right\rangle$ and $\left|\phi_{b}\right\rangle$, that are defined by diabatic Hamiltonians, $\hat{H}_{a}$ and $\hat{H}_{b}$, respectively. For any given configuration of the classical subsystem, $\mathbf{x}(t)$, the Hamiltonian for the quantum subsystem can therefore be written as

$$
\hat{H}[\mathbf{x}(t)]=\hat{H}_{a}[\mathbf{x}(t)]+\hat{H}_{b}[\mathbf{x}(t)]+\hat{V}[\mathbf{x}(t)]
$$

where

$$
\begin{aligned}
& \hat{H}_{a}[\mathbf{x}(t)]=E_{\mathrm{a}}[\mathbf{x}(t)]\left|\phi_{a}\right\rangle\left\langle\phi_{a}\right| \\
& \hat{H}_{b}[\mathbf{x}(t)]=E_{b}[\mathbf{x}(t)]\left|\phi_{b}\right\rangle\left\langle\phi_{b}\right|
\end{aligned}
$$

and

$$
\hat{V}[\mathbf{x}(t)]=V_{a b}[\mathbf{x}(t)]\left|\phi_{a}\right\rangle\left\langle\phi_{b}\left|+V_{a b}[\mathbf{x}(t)]\right| \phi_{b}\right\rangle\left\langle\phi_{a}\right|
$$

In these equations, $E_{a}$ and $E_{b}$ are the energies of the respective diabatic states, and $V_{a b}$ is the coupling magnitude between the diabats.

Under the Born-Oppenheimer approximation, the eigenstates and eigenvalues of the quantum subsystem can be solved by diagonalizing the full Hamiltonian. In order for the diabatic coupling to be physically meaningful, the diabatic states must be orthogonal. ${ }^{17}$ For orthonormal diabatic states, the adiabatic energy gap is related to the coupling strength by

$$
\lambda_{2}-\lambda_{1}=\sqrt{\left(E_{\mathrm{a}}-E_{b}\right)^{2}+4 V_{a b}^{2}}
$$

At a crossing point (i.e., $E_{a}=E_{b}$ ), the left-hand side of eq 7 is minimized and the intrinsic value of $V_{a b}$ can be determined. However, since the quantities in eq 7 generally depend on $\mathbf{x}(t)$, a minimum in $\lambda_{2}-\lambda_{1}$ does not necessarily correspond to a crossing point. We thus base our identification of crossing points on the diabatic composition of the adiabatic wave function. Specifically, the eigenstates of the full Hamiltonian in eq 3 have the general form ${ }^{18}$

$$
\begin{aligned}
& \left|\Psi_{1}(t)\right\rangle=c_{a}^{(1)}(t)\left|\phi_{a}\right\rangle+c_{b}^{(1)}(t)\left|\phi_{b}\right\rangle \\
& \left|\Psi_{2}(t)\right\rangle=c_{a}^{(2)}(t)\left|\phi_{a}\right\rangle+c_{b}^{(2)}(t)\left|\phi_{b}\right\rangle
\end{aligned}
$$

where $\left|c_{a}^{(\alpha)}(t)\right|^{2}+\left|c_{b}^{(\alpha)}(t)\right|^{2}=1$. The coefficients in the equations above can be determined through a unitary transformation of $\hat{H}$ (see the Supporting Information for more details) and expressed as simple functions of the mixing angle

$$
\tau[\mathbf{x}(t)]=\arctan \frac{2 V_{a b}[\mathbf{x}(t)]}{E_{\mathrm{a}}[\mathbf{x}(t)]-E_{b}[\mathbf{x}(t)]}
$$

Notably, at a crossing point, $\tau= \pm \pi / 2$, corresponding to the case where each adiabatic state contains an equal mixture of $\left|\phi_{a}\right\rangle$ and $\left|\phi_{b}\right\rangle$.

We distinguish between avoided and trivial crossings on the basis of the strength of the intrinsic coupling, $V_{a b}$, evaluated at the crossing point. We identify crossing points on the basis of the diabatic composition of the adiabatic wave functions. Specifically, we evaluate the joint probability density between eigenstates 1 and 2

$$
P_{1,2}[\mathbf{x}(t)]=\int \rho_{1}(\mathbf{r} ; \mathbf{x}(t)) \rho_{2}(\mathbf{r} ; \mathbf{x}(t)) \mathrm{d} \mathbf{r}
$$

where $\rho_{i}(\mathbf{r})=\left|\Psi_{i}(\mathbf{r})\right|^{2}$ and the integral is carried out over all space. The quantity $P_{1,2}$ depends on the configuration of the classical subsystem and thus varies in time along a trajectory. As a system enters a crossing and the eigenstates adopt complementary superposition states, the value of $P_{1,2}$ increases to a local maximum when $\tau \approx \pm \pi / 2$, i.e., the eigenstates are maximally overlapping. We assume that this local maximum corresponds to the crossing point and thus defines the crossing time, $t_{\mathcal{O}}$ as the point in time where $P_{1,2}$ is at a local maximum. Likewise, $P_{1,2}$ will go through another local maximum if the two states recross. We denote the time point of recrossing as $t_{\mathrm{r}}$. At the crossing point, we compute $V_{a b}$ using eq 6 and categorize the point as an avoided crossing, if $\left|V_{a b}\right| \geq V_{\mathcal{c}}$, or a trivial crossing, if $\left|V_{a b}\right|<V_{c}$. The value of $V_{c}$ can be determined on the basis of theoretical models, such as the Landau-Zener formula, ${ }^{18}$ or fit to match experimental data, as we describe in more detail in sections III and IV below.

Once a trivial crossing is identified, we correct the TCP by temporarily modifying the Hamiltonian to effectively eliminate the higher energy (crossing) diabatic state. There are numerous ways to perform such a modification, for example, by incorporating a diabatic switching function into the original Hamiltonian

$$
\hat{H}_{\text {mod }}(t)=\hat{H}_{a}+f_{b}(t)\left[\hat{H}_{b}+\hat{V}_{a b}\right]
$$

where the switching function is defined as

$$
f_{b}(t)= \begin{cases}0 & \text { if }\left|V_{a b}\right| \leq V_{\mathrm{c}} \text { and } t_{\mathrm{c}} \leq t \leq t_{\mathrm{r}} \\ 1 & \text { otherwise }\end{cases}
$$

The switching function $f_{b}$ eliminates the influence of the diabatic state $\left|\phi_{b}\right\rangle$ during the trivial crossing period, starting from the trivial crossing point $\left(t=t_{\mathrm{c}}\right)$ and ending at the trivial recrossing point $\left(t=t_{\mathrm{r}}\right)$. The evolution of the classical subsystem then proceeds according to the forces derived from the lowest energy eigenstate of the modified Hamiltonian in eq 12 (i.e., $\left|\Psi_{1}^{(\bmod )}\right\rangle$ ) and from the molecular mechanics force field. Notably, the modified Hamiltonian has the property that $E_{1}^{(\mathrm{mod})} \approx E_{1}$ at $t=t_{\mathrm{c}}$ and $t_{\mathrm{r}},\left|\left\langle\Psi_{1}^{(\mathrm{mod})}(t) \mid \Psi_{1}(t)\right\rangle\right| \approx 0.5$ at $t=t_{\mathrm{c}}$ and $\left|\left\langle\Psi_{1}^{(\text {mod })}(t) \mid \Psi_{1}(t)\right\rangle\right| \approx 1$ at $t=t_{\mathrm{r}}$.

This type of two-state solution can be generally applied to more complicated many-state systems provided that the diabatic wave functions and their coupling term do not change significantly between the trivial crossing and recrossing points. If diabatic states do undergo significant changes during the trivial crossing interval (i.e., $t_{\mathrm{c}}<t<t_{\mathrm{r}}$ ), then it must be verified that the coupling (e.g., $V_{a b}$ ) remains below the threshold during the entire time interval. If this is not the case, then purely adiabatic dynamics is probably inappropriate for describing the evolution of the quantum subsystem. For multistate systems, this approach can be applied recursively to address trivial crossings that occur within the dynamics of the modified Hamiltonian. In this case, the modified Hamiltonian can itself be modified, as shown in the Supporting Information.

II.B. Solutions for the Tight-Binding Model. In this model, the quantum subsystem is expressed using a Hamiltonian of the form 


$$
\hat{H}[\mathbf{x}(t)]=\sum_{i} \varepsilon_{i}[\mathbf{x}(t)]|i\rangle\left\langle i\left|+\sum_{i \neq j} J_{i j}[\mathbf{x}(t)]\right| i\right\rangle\langle j|
$$

where $|i\rangle$ represents a member of an orthonormal timeindependent basis, $\varepsilon_{i}$ is the energy of the system in basis state I $i\rangle$, and $J_{i j}$ is the coupling between basis states $|i\rangle$ and $|j\rangle$. As the notation indicates, in a mixed quantum-classical simulation, both $\varepsilon_{i}$ and $J_{i j}$ generally depend on the state of the classical subsystem. For quantum subsystems that adopt this common form, it is convenient to define diabatic states in the same basis as the original Hamiltonian, i.e.,

$$
\begin{aligned}
& \left|\phi_{a}\right\rangle=\sum_{i} c_{i}^{(a)}|i\rangle \\
& \left|\phi_{b}\right\rangle=\sum_{i} c_{i}^{(b)}|i\rangle
\end{aligned}
$$

where the coefficients define the subset of basis states that make up the diabat and $c_{i}^{(b)}=0$ if $c_{i}^{(a)} \neq 0$ and vice versa. Likewise, the diabatic Hamiltonian can be defined as

$$
\hat{H}_{a}=\sum_{i \in a} \varepsilon_{i}|i\rangle\left\langle i\left|+\sum_{(i \neq j) \in a} J_{i j}\right| i\right\rangle\langle j|
$$

where the summations only include those basis states with nonzero values of $c_{i}^{(a)}$. An analogous diabatic Hamiltonian can be generated for $\hat{H}_{b}$, so that the coupling operator takes the form

$$
V_{a b}=\sum_{i \in a} \sum_{j \in b}\left(J_{i j}|i\rangle\left\langle j\left|+J_{j i}\right| j\right\rangle\langle i|\right)
$$

With this formulation, the full Hamiltonian can be expressed in terms of the two lowest energy diabatic Hamiltonians, $\hat{H}_{a}$ and $\hat{H}_{b}$, their coupling operator, $\hat{V}_{a b}$, and a third effective diabatic state, $c$, representing the remainder of the quantum subsystem, i.e.,

$$
\hat{H}=\hat{H}_{a}+\hat{H}_{b}+\hat{V}_{a b}+\left(\hat{H}_{c}+\hat{V}_{a c}+\hat{V}_{b c}\right)
$$

where the basis states associated with diabat $c$ are all of those not included in either diabat $a$ or $b$. In this notation, the effective diabat $c$ may contain multiple eigenstates of the original Hamiltonian, while diabats $a$ and $b$ each correspond to a single eigenstate. By construction, these diabatic states are nonoverlapping and the Hamiltonian decomposition in eq 19 can be expressed in matrix form

$$
\mathbf{H}=\left[\begin{array}{ccc}
\mathbf{H}_{\mathrm{a}} & \mathbf{V}_{\mathrm{ac}} & \mathbf{V}_{\mathrm{ab}} \\
\mathbf{V}_{\mathrm{ac}} & \mathbf{H}_{\mathrm{c}} & \mathbf{V}_{\mathrm{bc}} \\
\mathbf{V}_{\mathrm{ab}} & \mathbf{V}_{\mathrm{bc}} & \mathbf{H}_{\mathrm{b}}
\end{array}\right]
$$

The relationship between the diabatic and adiabatic representations is illustrated in Figure 2a,b.

To prevent trivial crossings in a tight binding Hamiltonian model, we apply the switching function algorithm (eq 12). In this method, the basis states associated with diabat $b$ are removed from the Hamiltonian in eq 14 during the trivial crossing period to eliminate the influence of the crossing diabat $b$, as illustrated in Figure 2c. In addition to the switching function algorithm, another strategy for modifying the Hamiltonian is to artificially shift the energy level of the crossing diabatic state, as depicted in Figure 2d. This strategy is convenient to implement in adiabatic simulations because the size of the quantum subsystem is preserved upon switching a) Diabatic picture

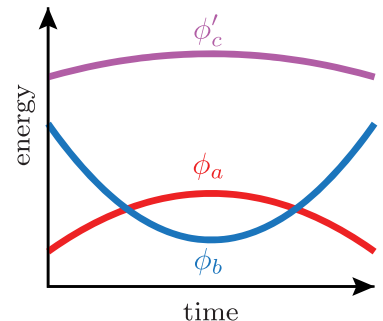

c) Switching function

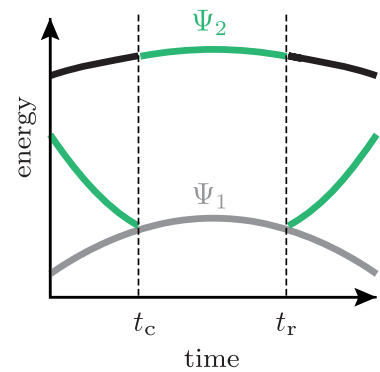

b) Adiabatic picture

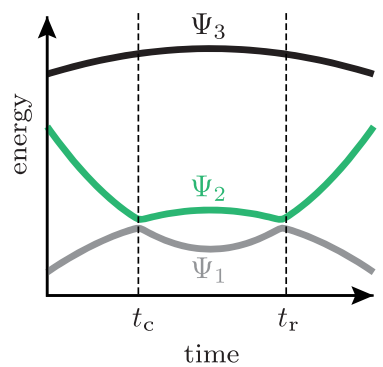

d) Energetic bias

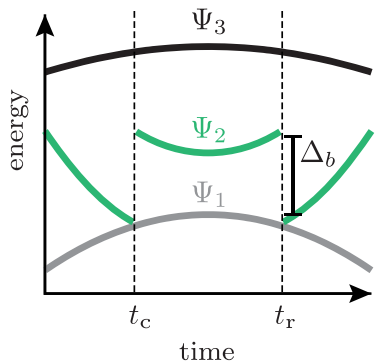

Figure 2. Schematic of a three-state system showing a trivial crossing problem and its proposed solutions. (a) Energy level crossings in diabatic basis. Here $\phi_{c}^{\prime}$ represents the lowest eigenvalue of the effective diabat $c$ (see text for more details). (b) The same system as that in panel a but in the adiabatic basis. In this example, since the coupling between states $a$ and $b$ is negligible, this case presents a TCP. Panels $c$ and $d$ show the consequences of our solution to the TCP in the adiabatic basis. Panel $\mathrm{c}$ shows the effect of eliminating the crossing diabat, as in eq 12. Panel d shows the effect of shifting the eigenvalue of the crossing diabat, as in eq 21.

between the standard and modified Hamiltonians. Formally, the modified Hamiltonian for this approach is given by the form

$$
\hat{H}_{\text {mod }}=\hat{H}+\sum_{i \in b} \Delta_{b}|i\rangle\langle i|
$$

where $\Delta_{b}$ is a constant that is large enough to eliminate the crossing of diabats $a$ and $b$ along the entire time interval $t_{\mathrm{c}} \leq t$ $\leq t_{\mathrm{r}}$.

\section{APPLICATION: EXCITON DYNAMICS ON EXTENDED CONJUGATED POLYMER SYSTEMS}

The energy transport properties of electronically excited conjugated polymers contribute significantly to the performance of organic electronic applications, such as organic photovoltaic and light-emitting devices. ${ }^{19-21}$ One of the challenges in modeling excited state properties is that these systems tend to exhibit frequent trivial crossings, as first discovered by Fernandez-Alberti et al. for phenylene ethynylene oligomer chains. ${ }^{2}$ Furthermore, the frequency of trivial crossings in these systems increases with polymer chain length, making it difficult to simulate anything but short (e.g., $\sim 10$ monomer units) isolated oligomers. A robust and accurate solution to the TCP is therefore essential to enabling the simulation of experimentally relevant time and length scales.

The tendency of these systems to exhibit TCPs arises because these systems possess a manifold of low-lying electronic excited states that are easily localized to nanometer 
length scales in the presence of room temperature thermal disorder. When these states are spatially separated, they exhibit weak coupling and can thus contibute to TCPs. For extended conjugated polymers on their ground-state potential energy surface, the low lying excited states occupy the same crowded band of energies. This band includes numerous avoided and trivial crossings. By contrast, when the system is on an excitedstate potential energy surface, the active eigenstate can be preferentially stabilized via polaronic interactions between the classical and quantum subsystems. These interactions helps reduce the energy of the active state and the number of state crossings.

Polaronic effects in neutral conjugated polymer systems arise due to the interaction of an electronic excitation, also known as an exciton, with the nuclear degrees of freedom. These interactions favor the planarization of the monomer-monomer torsional landscape. ${ }^{22}$ This planarization results in a higher degree of $\pi$-conjugation and thus a lowering of the excited state energy level. ${ }^{23}$ Since these planarizing forces are colocalized with the position of the exciton, only a limited region of the polymer chain is influenced, resulting in a phenomenon known as exciton self-trapping. ${ }^{24-27}$ The dynamics of self-trapped excitons is thus naturally described in diabatic states that are spatially localized over several monomer units.

III.A. Model Description. The dynamics of an electronically excited conjugated polymer system can be efficiently modeled using a mixed quantum/classical approach in which the quantum subsystem is described by a Frenkel exciton Hamiltonian. ${ }^{28}$ In the Frenkel exciton Hamiltonian, the excited states of a polymer with $N$ monomers are expressed in an orthonormal basis of single monomer excitations, with a Hamiltonian of the form

$$
\hat{H}_{\text {Frenkel }}=\sum_{i}^{N} \varepsilon_{i}|i\rangle\left\langle i\left|+\sum_{i \neq j} J_{i j}\right| i\right\rangle\langle j|
$$

where $|i\rangle$ represents the state in which only the $i$ th monomer is excited (all other monomers are thus assumed to be in the ground state), $\varepsilon_{i}$ is the associated monomer excitation energy, and $J_{i j}$ is the electronic coupling between states $|i\rangle$ and $|j\rangle$. In the results presented below, we adopt a variation of this model that is specific to the physics of conjugated polymer systems and similar to that developed by Tozer and Barford to model poly(para-phenylene) chains. ${ }^{11}$ In this variation, the monomer excitation energies are all assumed to be identical, given by the parameter $\epsilon_{0}$, and the coupling $J_{i j}$ is assumed to be the sum of a through-bond and a through-space contribution. The Hamiltonian of our model is given by

$$
\begin{aligned}
\hat{H}_{\text {poly }}= & \sum_{i}^{N} \varepsilon_{0}|i\rangle\left\langle i\left|+\sum_{i \neq j}^{N} J_{i j}^{(\text {dip })}\right| i\right\rangle\langle j| \\
& +\sum_{i}^{N-1} J_{i}^{(\text {bond })}(|i\rangle\langle i+1|+| i+1\rangle\langle i|)
\end{aligned}
$$

where $J_{i j}^{(\text {dip })}$ describes the through-space dipolar coupling of states $|i\rangle$ and $|j\rangle$ and $J_{i}^{(\text {bond })}$ describes the through-bond coupling of adjacent monomers. Specifically, the through-space coupling is given by

$$
J_{i j}^{(\mathrm{dip})}=\frac{\kappa_{i j} \mu_{0}^{2}}{4 \pi \epsilon_{\mathrm{r}} \epsilon_{0} R_{i j}^{3}}
$$

where $\mu_{0}$ is the strength of the transition dipole of state $|i\rangle, \epsilon_{\mathrm{r}}$ and $\epsilon_{0}$ denote the dielectric constant and the vacuum permittivity, respectively, $R_{i j}=\left|\mathbf{R}_{i j}\right|$ is the separation between monomer units $i$ and $j$, and $\kappa_{i j}=\hat{\mu}_{i} \cdot \hat{\mu}_{j}-3\left(\mathbf{R}_{i j} \cdot \hat{\mu}_{i}\right)\left(\mathbf{R}_{i j} \cdot \hat{\mu}_{j}\right) / R_{i j}{ }^{2}$ is the dipole orientation factor, where $\hat{\mu}_{i}$ is the unit dipole vector for the transition dipole of state $|i\rangle .{ }^{29}$ The through-bond coupling is given by

$$
J_{i}^{(\text {bond })}=J_{\text {SE }} \cos ^{2}\left(\theta_{i}\right)
$$

where $J_{\mathrm{SE}}$ is a constant determined by the electronic exchange coupling between bonded monomers and $\theta_{i}$ is the torsional angle between monomers $i$ and $(i+1)$.

For the classical subsystem, we utilize a coarse grained model of a polymer that only includes the degrees of freedom that have dependencies in the quantum Hamiltonian of eq 23. Namely, this includes the relative positions of monomers and the set of monomer-monomer torsional angles. In this coarse grained model, we describe the dynamics of the torsional landscape via a Langevin equation

$$
I \frac{\mathrm{d}^{2} \theta_{i}}{\mathrm{~d} t^{2}}=-\eta \frac{\mathrm{d} \theta_{i}}{\mathrm{~d} t}-\frac{\mathrm{d} U_{\mathrm{g}}\left(\theta_{i}\right)}{\mathrm{d} \theta_{i}}+F_{\mathrm{ex}}^{(n)}\left(\theta_{i}\right)+\xi_{i}(t)
$$

where $I$ is the moment of inertia of a monomer ring about a rotational axis parallel to the polymer backbone, $\eta$ is the friction coefficient for a given solvent, $U_{\mathrm{g}}(\theta)$ is the ground state torsional potential energy function, $F_{\mathrm{ex}}^{(n)}$ is the torque exerted on monomer rings by the $n$th exciton state, and $\xi$ is the stochastic torque on monomers due to the random fluctuations in the solvent. We model these random fluctuations as white noise with a correlation function, $\left\langle\xi_{i}(t) \xi_{j}(t+\delta t)\right\rangle=$ $2 \eta k_{\mathrm{B}} T \delta_{i j} \delta(\delta t)$, where $k_{\mathrm{B}} T$ is the Boltzmann constant times temperature. In this work, the ground state potential energy function, $U_{\mathrm{g}}(\theta)$, was numerically tabulated on the basis of the analysis of all-atom molecular dynamics simulation data, as described in more detail in the following subsection.

We determine the difference between the ground state and the excited state forces from the Hellmann-Feynman theorem

$$
\begin{aligned}
F_{\mathrm{ex}}^{(n)}\left(\theta_{i}\right) & =-\left\langle\Psi_{n}\left|\frac{\partial \hat{H}_{\mathrm{ex}}}{\partial \theta_{i}}\right| \Psi_{n}\right\rangle \\
& =2 J_{\mathrm{SE}} \sin \left(2 \theta_{i}\right) b_{i}^{(n)} b_{i+1}^{(n)}
\end{aligned}
$$

where $b_{i}^{(n)}=\left\langle i \mid \Psi_{n}\right\rangle$. Notably, this expression for the excited state force vanishes in regions of the polymer that are far from the position of the localized exciton. We assume that there is a separation of time scales between the lifetime of an exciton and the changes in the spatial configuration of the polymer. Thus, we treat the relative monomer positions as static. In this work, we assume the polymer is in an idealized extended linear configuration unless described otherwise.

III.B. Model Parameterization. We parametrize our model to correspond to simulation results generated for polythiophene in nonpolar solvent at $T=300 \mathrm{~K}$. The parameters of the quantum Hamiltonian are assigned on the basis of mixed QM/MM excited-state all-atom molecular dynamics (MD) simulations. These simulations utilize a QCFF/PI approach, ${ }^{30}$ similar to one originally developed by Warshel and Karplus. ${ }^{31}$ In this approach, the electronic structure is only explicitly evaluated for the $\pi$-electrons, which are treated using a semiempirical Pariser-Parr-Pople (PPP) type Hamiltonian. ${ }^{32-34}$ All other electronic degrees of 
freedom are treated implicitly in the parametrization of the molecular mechanics force field. Excited state properties are computed using configuration interaction with single excitations (i.e., CIS).

Following the specific approach of refs 35 and 36, we generate multiple independent trajectories of a 30-mer thiophene at $T=300 \mathrm{~K}$ in the first electronically excited state. This method is described in more detail in the Supporting Information. Shorter quaterthiophene oligomers are simulated in the ground state to compute the ground state torsional potential energy, $U_{\mathrm{g}}(\theta)$, used in eq 26. $U_{\mathrm{g}}(\theta)$ is tabulated to reproduce the potential of mean force for ringring torsional angles computed from atomistic quaterthiophene simulations, and the resulting potential is described in the Supporting Information.

This ground state torsional potential is bimodal with a local minimum at $35^{\circ}$ (cis-configuration) and a global minimum at $143^{\circ}$ (trans-configuration) that are separated by a barrier of approximately $12 \mathrm{meV}$. We assume that torsional angles are independent, which is supported by correlation analysis of the MD simulations. We neglect the subtle differences in $U_{\mathrm{g}}(\theta)$ that emerge at the chain ends. Further details about the MD simulations can be found in the Supporting Information.

Table 1 lists the parameters we derive on the basis of the results of these simulations. These values are used to simulate

Table 1. Parameters of the Exciton Model of Conjugated Polymers

\begin{tabular}{cccc}
\multicolumn{5}{c}{ quantum } \\
\hline$\varepsilon_{0}(\mathrm{eV})$ & $J_{\mathrm{SE}}(\mathrm{eV})$ & $\epsilon_{\mathrm{r}}$ & $\mu_{0}(\mathrm{D})$ \\
5.0 & -0.5 & 2.7 & 9.0 \\
\multicolumn{4}{c}{ classical } \\
\hline$I\left(\mathrm{eV} \mathrm{fs}^{2} \mathrm{deg}^{-2}\right)$ & & \multicolumn{2}{c}{$\eta\left(\mathrm{eV} \mathrm{fs} \mathrm{deg}^{-2}\right)$} \\
\multicolumn{2}{c}{3.0} & \multicolumn{2}{c}{0.0035} \\
\hline
\end{tabular}

the motion of exciton in a polythiophene chain in nonpolar solvent at room temperature $(T=300 \mathrm{~K})$.

III.C. Model Implementation. Using our coarse grained model, we consider the time evolution of the exciton wave function starting in the lowest energy excited state. Before initializing our exciton dynamics, we prepare a polythiothene chain with $\mathrm{N}$-monomers whose centroid positions are uniformly spaced by $3.88 \AA$ along the $x$-axis. The initial torsional angles and their velocities are randomly drawn from a uniform distribution between $-180^{\circ}$ and $180^{\circ}$ and from the Maxwell-Boltzmann distribution at $T=300 \mathrm{~K}$, respectively. We first equilibrate the ground state torsional angle configuration for 10 ps. The system is then evolved along the potential energy surface of the lowest eigenstate $(n=1)$ via eq 26. We numerically integrate eq 26 using the method developed by Vanden-Eijnden and Ciccotti ${ }^{37}$ with a time step of $\delta t=1$ fs. All results presented in this paper have been insensitive to the integration time step as long as $\delta t \leq 1$ fs.

During adiabatic dynamics, we identify crossing points by evaluating the joint probability density, $P_{1,2}$, as described in section II.A. We assume that, at a crossing point, $\left|\Psi_{1}\right\rangle$ and $\left|\Psi_{2}\right\rangle$ represent spatially symmetric and antisymmetric linear combinations of diabatic states $\left|\phi_{a}\right\rangle$ and $\left|\phi_{b}\right\rangle$ (see eqs 8 and 9). We diabatize the quantum subsystem on the basis of this assumption by locating the node in $\left|\Psi_{2}\right\rangle$ and then constructing two orthonormal diabatic states that are constrained to lie on opposite sides of the node. We set $\left|\phi_{a}\right\rangle\left(\right.$ or $\left.\left|\phi_{b}\right\rangle\right)$ to be the diabatic wave functions with bigger (or smaller) overlap with the active state prior to the crossing point, $\left|\Psi_{1}\left(t_{c}-\delta t\right)\right\rangle$. The resulting diabats can thus be represented in a block matrix form similar to that of eq 20 .

We compute the electronic coupling between the diabatic states under a line dipole approximation ${ }^{38}$

$$
V_{a b}=\left\langle\phi\left|\hat{V}_{a b}\right| \phi_{b}\right\rangle=\frac{\mu_{0}{ }^{2}}{4 \pi \epsilon_{\mathrm{r}} \epsilon_{0}} \sum_{i}^{N} \sum_{j \neq i}^{N} \frac{\kappa_{i j}}{R_{i j}{ }^{3}} c_{i}^{(a)} c_{j}^{(b)}
$$

where $c_{i}^{(a)}=\left\langle i \mid \phi_{a}\right\rangle$ and $c_{j}^{(b)}=\left\langle j \mid \phi_{b}\right\rangle$.

We perform adiabatic dynamics along the lowest potential energy surface of the exciton Hamiltonian and use the joint probability density (eq 11) and the switching function algorithm (eq 12) described in section II to identify and prevent trivial crossings. We define the recrossing point to be when $\left|\left\langle\Psi_{1}^{(\mathrm{mod})}(t) \mid \Psi_{1}(t)\right\rangle\right|=S_{\mathcal{c}}$, where $S_{\mathrm{c}}$ equals 1 in theory. However, in practice, it is less than 1 due to discrete time steps and floating point error during the evaluation of the overlap integral. In this conjugated polymer example, we have chosen this recrossing threshold value to be $S_{\mathrm{c}}=0.99$, though any value greater than 0.7 works essentially the same without changing the adiabatic dynamics (see Figure S1). The algorithm we employ in these simulations is illustrated in the form of a flowchart in Figure 3.

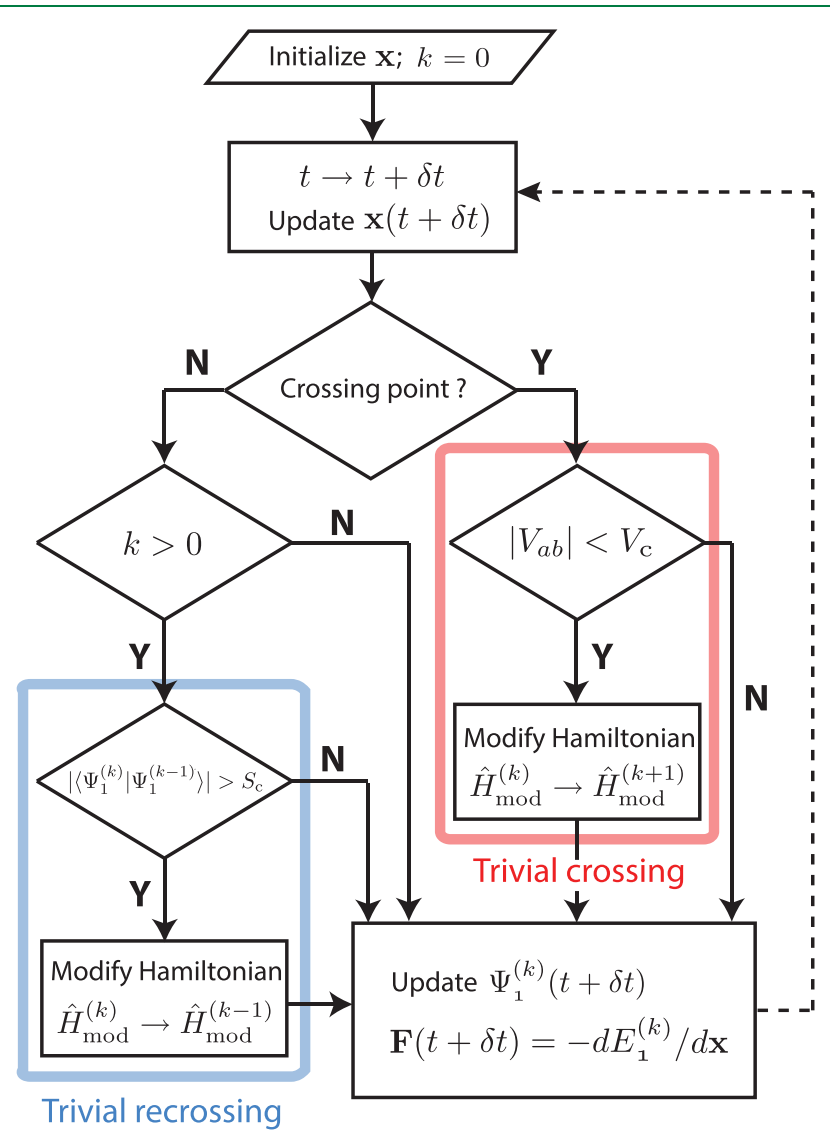

Figure 3. Algorithm flowchart for the symmetry-preserving adiabatic dynamics. Here $k$ is defined as the number of recursive modifications made to the original Hamiltonian $\hat{H}$ in the response to trivial crossings. In this notation, $\hat{H}_{\bmod }^{(0)}$ is equivalent to the original unmodified Hamiltonian. For the results presented in this paper, we use $S_{\mathrm{c}}=0.99$. 
In Figure 4a, we illustrate the outcome of our diabatization scheme for two eigenstates, $\Psi_{1}$ and $\Psi_{2}$, at their crossing point.
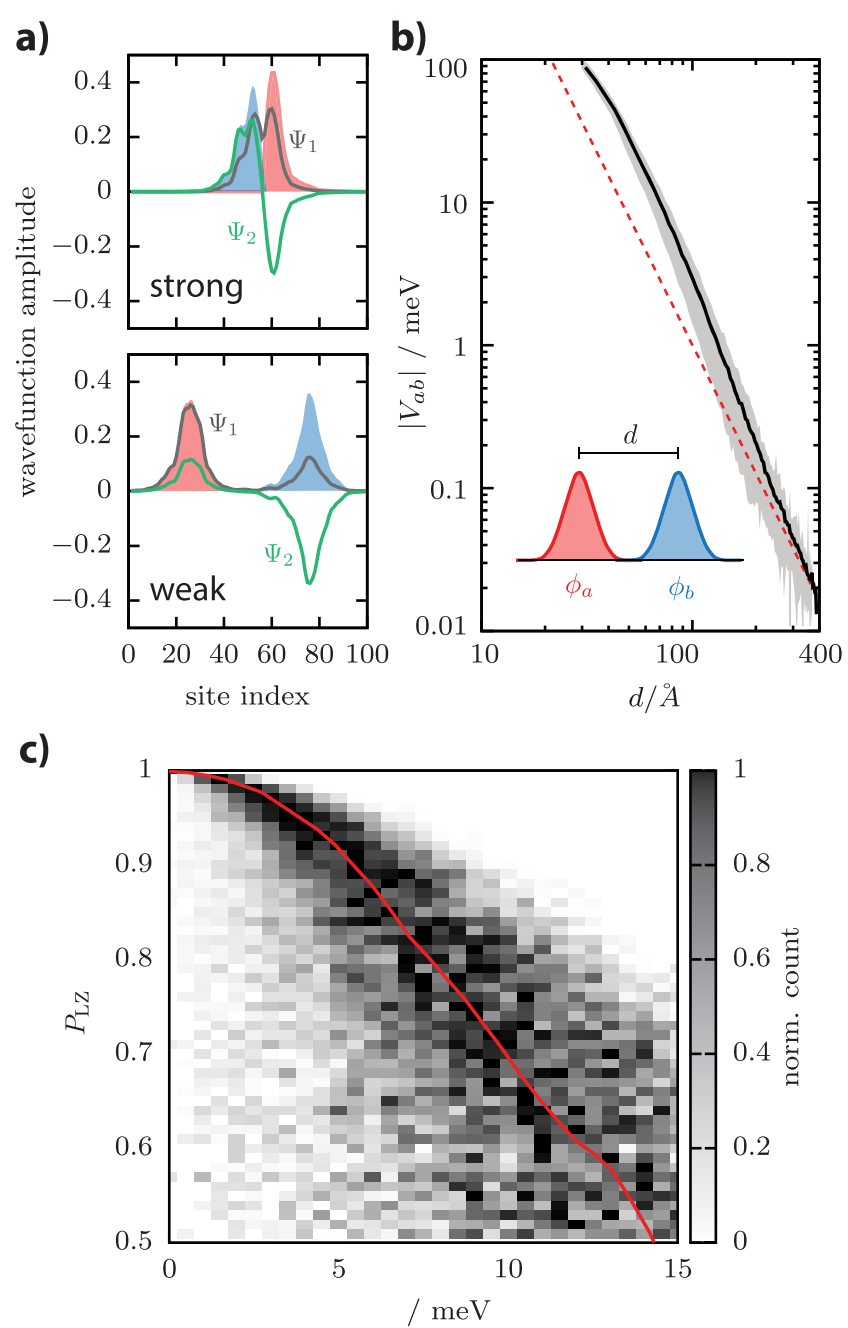

Figure 4. Electronic coupling between diabats of a 100-mer chain, computed from our model. (a) A snapshot of strong $\left(V_{a b}=50 \mathrm{meV}\right)$ versus weak $\left(V_{a b}=0.5 \mathrm{meV}\right)$ electronic coupling. The solid lines indicate adiabatic states $(1=$ gray, $2=$ green $)$ near the crossing points. The shaded curves represent diabatic states $\phi_{a}$ (red) and $\phi_{b}$ (blue). (b) Electronic coupling versus the distance between two diabatic wave functions. The average electronic coupling for a given distance is plotted in a black line, while the shaded gray region represents one standard deviation about the mean. The dotted red line shows the point-dipole approximation, $V \propto 1 / r^{3}$. (c) The relationship between the Landau-Zener transition probability, $P_{\mathrm{LZ}}$, and the diabatic coupling, $\left|V_{a b}\right|$. The red line indicates the average value of $P_{\mathrm{LZ}}$. The shading represents the number of data points falling inside each bin normalized by the maximum number for a given coupling value. In panels $\mathrm{b}$ and $\mathrm{c}$, the plotted data reflects statistics over approximately 4 $\times 10^{5}$ crossing time points sampled from 5000 independent trajectories.

The figure also contrasts the cases of strongly and weakly coupled states. Figure $4 \mathrm{~b}$ contains a plot of the average coupling between crossing diabatic states as a function of the distance between the diabats

$$
d_{a b}=\left|\left\langle\phi_{a}|\hat{x}| \phi_{a}\right\rangle\right|-\left|\left\langle\phi_{b}|\hat{x}| \phi_{b}\right\rangle\right|
$$

The coupling strength versus distance converges to a point dipole scaling, i.e., $V \propto 1 / r^{3}$, for $d>200 \AA$, corresponding to
50 or more monomer units. The variation in computed coupling strength, as indicated in shaded areas, stems from variations in the state of the classical subsystem (i.e., the ringring torsional landscape) within our sampled ensemble of crossing points.

While the implementation of our method adds to the baseline computational expense of a purely adiabatic simulation, it is less costly than performing a standard nonadiabatic simulation. Even the most efficient nonadiabatic methods, such as those based on surface hopping, ${ }^{39}$ require the generation of an ensemble of individual trajectories, potentially increasing the computational scaling significantly relative to a single purely adiabatic simulation with our method. In addition, nonadiabatic techniques that use discrete time steps can straddle a trivial crossing and thus miss its impulsive influence altogether. Preventing this straddling effect therefore necessitates its own solutions, such as adaptive time stepping. ${ }^{40}$ It is worth noting that in cases where purely adiabatic dynamics is unreliable, such as in systems with dense and highly coupled manifolds of states, our approach is likely unreliable.

III.D. Selecting the Coupling Cutoff, $V_{c}$. We categorize crossing points as trivial or not on the basis of the computed value of the diabatic coupling, $\left|V_{a b}\right|$. Specifically, we identify a trivial crossing as having a value of $\left|V_{a b}\right|<V_{c}$ and a standard avoided crossing as having a value of $\left|V_{a b}\right| \geq V_{c}$. Standard Born-Oppenheimer adiabatic dynamics corresponds to the case where $V_{c}=0$, while the limit of large $V_{c}$ corresponds to a type of diabatic dynamics, albeit potentially unphysical.

One way to designate the value of $V_{c}$ is through the Landau-Zener formula ${ }^{18}$

$$
P_{\mathrm{LZ}}=\exp \left[\frac{-2 \pi V_{a b}^{2} / \hbar}{\left|\frac{\partial}{\partial t}\left(E_{b}-E_{\mathrm{a}}\right)\right|}\right]
$$

which specifies the nonadiabatic transition probability for a two-state system. In Figure 4c, we plot a histogram of the values of $P_{\mathrm{LZ}}$ and the corresponding values of $\left|V_{a b}\right|$ as computed from an equilibrium ensemble of configurations from our model. We observe significant scatter in the data but identify the expected trend that $P_{\mathrm{LZ}}$ increases with decreasing I $V_{\mathrm{ab}}$ l. This trend suggests the tendency of the system to exhibit nonadiabatic effects in response to a weakly coupled state crossing. We utilize this trend to assign a value of $V_{c}$. In particular, we choose $V_{\mathrm{c}}=5 \mathrm{meV}$, corresponding to the coupling value that has a Landau-Zenner transition probability of about $95 \%$. We find that the qualitative results of our findings presented in the rest of the paper do not depend on $V_{\mathrm{c}}$ as long as it is chosen such that $P_{\mathrm{LZ}} \gtrsim 0.7$.

\section{DEMONSTRATING THE PERFORMANCE OF OUR APPROACH}

In this section, we consider the dynamics of an excited state conjugated polymer system and show that our solution to the TCP yields physically reasonable adiabatic dynamics while preserving the nodeless symmetry of the excitonic wave function. We have used the switching function algorithm (eq 12) to demonstrate our trivial crossing correction approach, though the energy shifting method (eq 21) works equally well (see Figure S2). Specifically, we compare adiabatic trajectories of the lowest exciton state of a thiophene 200-mer initiated in identical states but with three different methods: the standard 


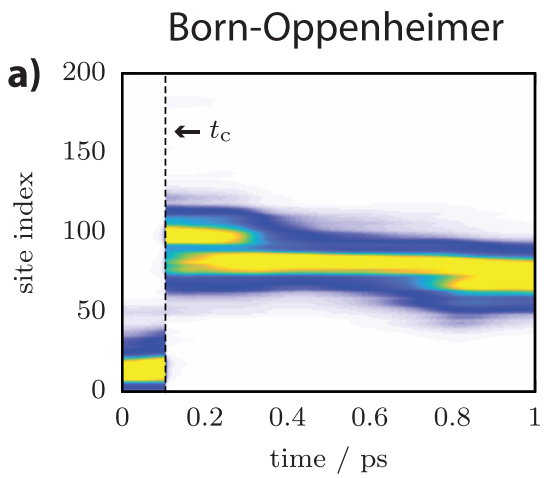

Non-symmetry-preserving

b)

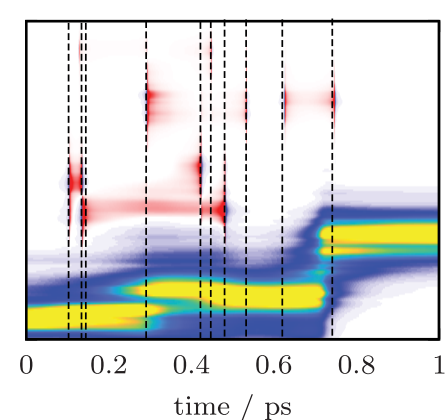

e)

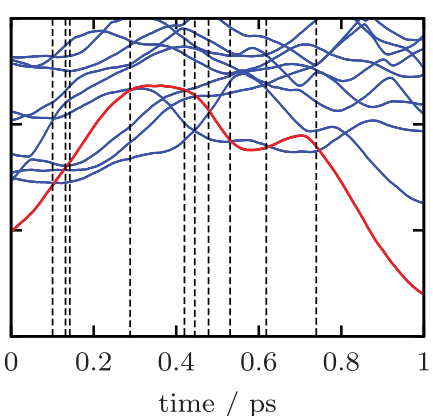

h)
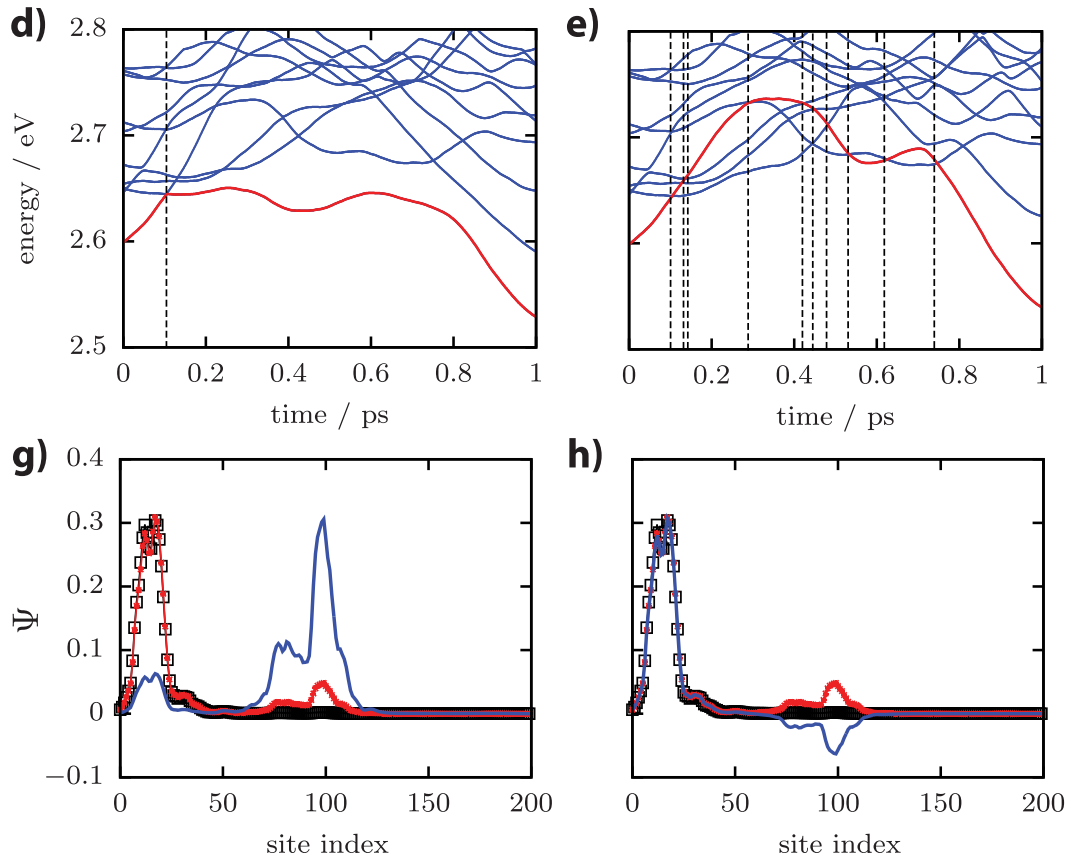

Symmetry-preserving

c)

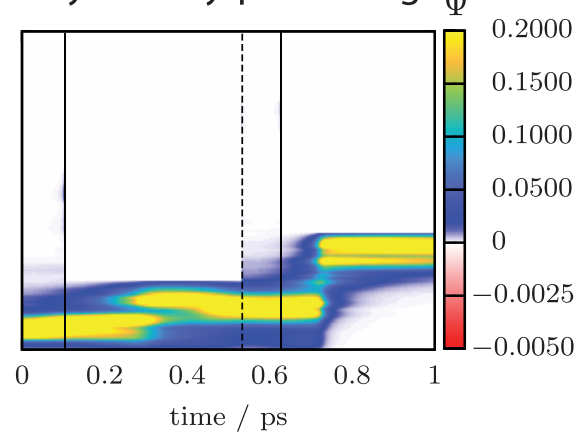

f)

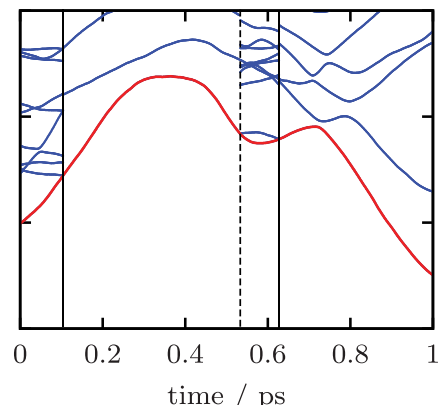

i)

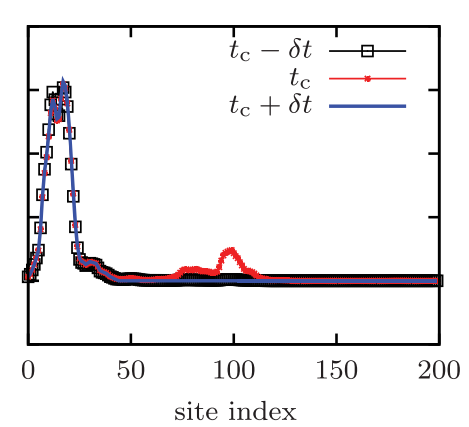

Figure 5. Comparing adiabatic dynamics from our symmetry-preserving method with the standard uncorrected Born-Oppenheimer and the nonsymmetry-preserving methods for a trajectory initialized from identical configurations. $(a-c)$ Shading corresponds to the wave function of the active adiatabic state. (a) In the Born-Oppenheimer dynamics, the active state is always the lowest eigenstate of the original Hamiltonian. The dashed vertical line marks a trivial crosssing point, $t_{\mathrm{c}}=0.1 \mathrm{ps}$, indicated by discontinuities in exciton position. (b) In the non-symmetry-preserving method, the active state hops between different adiabatic states, whose hopping time points are marked by dashed lines. (c) In the symmetrypreserving method, the active state is the lowest eigenstate of the modified Hamiltonian. A coupling cutoff, $V_{\mathrm{c}}$, of $5 \mathrm{meV}$ is used. Solid vertical lines mark the time when the Hamiltonian is modified in response to a trivial crossing. The dashed line indicates the point when the trivial crossing problem vanishes and the Hamiltonian is restored to its original unmodified form. (d-f) Time dependence of the excited state energy levels of the lowest eigenstates. The red curve indicates the active eigenstate, which determines the potential energy surface used to propagate adiabatic dynamics. ( $\mathrm{g}-\mathrm{i}$ ) Snapshots of the active state wavefuntion at the vicinity of the first trivial crossing, $t_{\mathrm{c}}=0.1 \mathrm{ps}$, with $\delta t=1 \mathrm{fs}$. While wave functions before and at the trivial crossing (black and red curves) are the same, resulting wave functions (blue curves) are different for all three methods once they pass through the trivial crossing.

Born-Oppenheimer-type adiabatic dynamics, our symmetrypreserving method, and a common non-symmetry-preserving approach also known as the overlap method. In the overlap method, the active adiabatic state for any given time step is selected to yield maximal wave function overlap with the active state of the previous time step. In this case, overlap between states $i$ and $j$ at adjacent time steps is given by

$$
S_{i, j}=\left|\left\langle\Psi_{i}(t) \mid \Psi_{j}(t+\delta t)\right\rangle\right|
$$

where $\delta t$ is the length of a single time step. As the active state hops between different eigenstates in the non-symmetrypreserving method, we conserve the phase of the active eigenstate as detailed in the Supporting Information. The results of these three trajectories are plotted in Figure 5.
We observe impulsive long-range exciton hopping in the Born-Oppenheimer dynamics case at $t \approx 0.1 \mathrm{ps}$, marked by a dashed line in Figure 5a and d. This long-range hopping event corresponds to a TCP, which is absent in both non-symmetrypreserving (Figure 5b) and symmetry-preserving (Figure 5c) methods. Notably, the TCP-free dynamics behaves identically as the Born-Oppenheimer dynamics until the point of trivial crossing (i.e., $t \approx 0.1 \mathrm{ps}$ ), where they evolve along differing adiabatic potential energy surfaces and thus begin to diverge. In the symmetry-preserving method, the lowest exciton state appears spatially unimodal unless it goes through an avoided crossing (e.g., at $t=0.3$ and $0.72 \mathrm{ps).}$

In our symmetry-preserving method and in BornOppenheimer dynamics, the active state is always a nodeless state, where the amplitude of the wave function remains 
positive throughout the entire trajectory, as illustrated in Figure 5a, c, g, and i. In the standard non-symmetry-preserving method, the nodal symmetry of the active wave function changes during the trivial crossing period, indicated by the appearance of negative amplitudes depicted in Figure 5b (red regions) and $\mathrm{h}$ (blue curve). For this particular example in Figure 5, the negative amplitudes are 1-2 orders of magnitude less than the positive ones, as noted by the color scale. Thus, Hellmann-Feynman forces, which are proportional to the active state wave function amplitude (eq 27), and the resulting nuclear configurations in both cases are nearly the same, as indicated by red curves in Figure 5e and $\mathrm{f}$. In general, however, we find that increasing the number of nodes leads to smaller magnitudes in Hellmann-Feynman forces. This leads to differences in adiabatic dynamics sampled using the nonsymmetry-preserving method compared to our symmetrypreserving scheme, as further discussed in section $\mathrm{V}$ below.

\section{THE EFFECT OF NODAL SYMMETRY PRESERVATION ON EXCITON TRANSPORT PROPERTIES}

We now apply our method for simulating wave function symmetry-preserving dynamics to study the migration of the lowest exciton state in long chain polythiophenes. We also demonstrate that failure to preserve the nodal symmetry of the adiabatic wave function can lead to unphysical exciton dynamics. We first compare the dynamics generated with our symmetry-preserving method to the dynamics generated with a non-symmetry-preserving method of solving the TCP. We characterize the dynamical properties of excitons in terms of their diffusion coefficients, which we quantify by computing exciton mean-squared-displacements (MSD), and their sizes. For a sample of $M$ independent exciton trajectories, MSD is defined as

$$
\operatorname{MSD}(t)=\frac{1}{M} \sum_{m}^{M}\left|x_{m}(t)-x_{m}(0)\right|^{2}
$$

where $x_{m}(t)=\left\langle\Psi_{1}^{(m)}(t)|\hat{x}| \Psi_{1}^{(m)}(t)\right\rangle$ is the centroid position of the exciton wave function at time $t$ for the $m$ th trajectory. Exciton sizes are quantified in terms of the inverse participation ratio (IPR)

$$
\operatorname{IPR}=\frac{1}{\sum_{i}^{N}\left\langle i \mid \Psi_{1}\right\rangle^{4}}
$$

which roughly corresponds to the number of basis states (i.e., excited monomers) that participate in a given excited state.

One contributing factor to differences between symmetrypreserving and non-symmetry-preserving dynamics is that the size of an adiabatic wave function generally increases with the number of wave function nodes. As such, the hopping between adiabatic states to prevent the TCP can lead to an unintended increase in wave function size. To illustrate this, we have computed the probability distribution of IPR values, $P$ (IPR), for each of the five lowest eigenstates of an ensemble of ground state torsional configurations of a model thiophene 100-mer. The results, plotted in Figure 6, illustrate that both the mean and the width of $P$ (IPR) increase with state index. In particular, the $n>1$ state features exaggerated high-IPR tails (Figure 6b) that reflect a greatly increased prevalence of highly delocalized states.
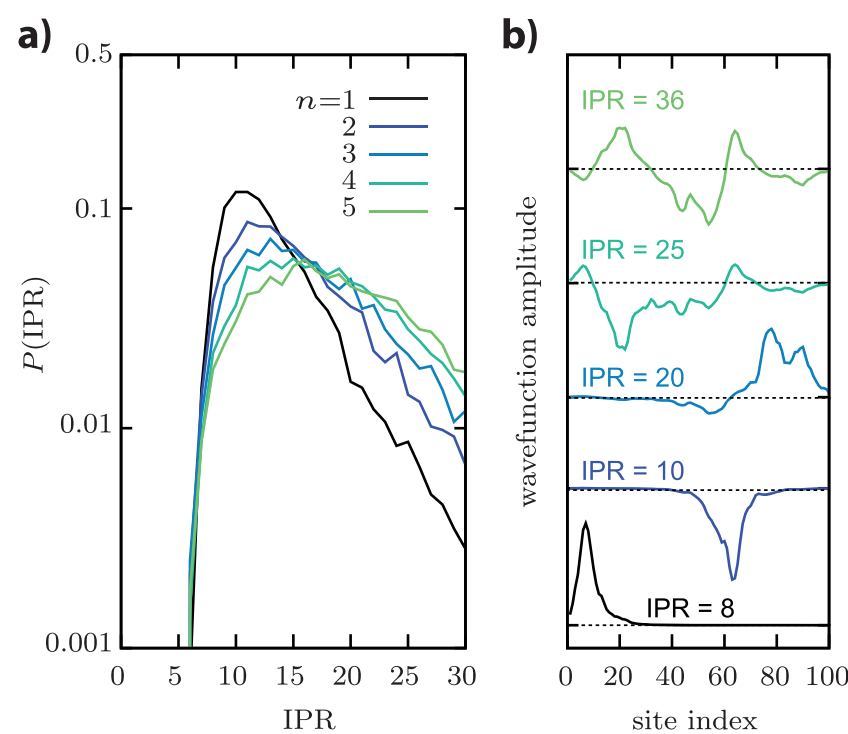

Figure 6. Dependence of exciton sizes on its eigenstate index. (a) Probability distribution for the IPR for the five lowest-energy wave functions, $n=1, \ldots, 5$. Distributions are computed over 500 equilibrium torsional configurations of a thiophene 100 -mer in the ground electronic state at $T=300 \mathrm{~K}$. (b) A snapshot of five lowestenergy adiabatic wave functions with their inverse participation ratios (IPRs) rounded to the nearest integer.

To illustrate the consequences of this effect, we compare the MSD of excitons generated using our symmetry-preserving approach to that generated with a non-symmetry-preserving method (eq 31). In all cases, the trajectories are initialized in the lowest energy excited state of a randomly selected equilibrium ground state configuration. To eliminate polymer chain end effects, we have implemented quenching boundary conditions that terminate dynamical trajectories if more than $90 \%$ of the exciton wave function is occupying the 15 terminal monomers on either end of the polymer chain. Quenched trajectories are included in the reported statistics and account for approximately $15 \%$ (for $N=300$ ) to $50 \%$ (for $N=100$ ) of overall trajectories with an average quenching time of $t_{\text {quench }} \approx$ 5 ps with $V_{\mathrm{c}}=5 \mathrm{meV}$.

The results plotted in Figure $7 \mathrm{a}$ and $\mathrm{b}$ show that the early time exciton diffusivity differs significantly between these two approaches to solving the TCP. The early time exciton diffusivity is given by the quantity $D_{\text {short }}=\operatorname{MSD}(t=1 \mathrm{ps}) /[2 \cdot 1$ ps], where the factor 2 in the denominator accounts for diffusion in one dimension. We find that $D_{\text {short }}$ for the nonsymmetry-preserving method yields larger values of the diffusion constant. Notably, the short time diffusivity of the non-symmetry-preserving approach grows with the length of the polymer chain. This length dependence is unexpected for chains with monomer numbers of $N \gtrsim\langle\mathrm{IPR}\rangle+\langle d\rangle / l_{0} \approx 40$, where $\langle\mathrm{IPR}\rangle$ is the average size of the diabat $(\langle\mathrm{IPR}\rangle \approx 8),\langle d\rangle$ is the average distance between diabats undergoing avoided crossings $\left(\langle d\rangle=150 \AA\right.$ for $\left.P_{\mathrm{LZ}} \approx 0.99\right)$, and $l_{0}$ is the distance between two thiophene rings $\left(l_{0}=3.88 \AA\right)$. The plateau in the MSD at longer times for the non-symmetry-preserving method represents a finite size effect.

Figure $7 \mathrm{~b}$ highlights that our symmetry-preserving approach delivers the desired physical behavior that exciton diffusivity is independent of chain length for sufficiently long polymer chains. For shorter chains $(N<200)$, the plateau in the MSD at longer times is due to a finite size effect. On the basis of the 
Non-symmetry-preserving
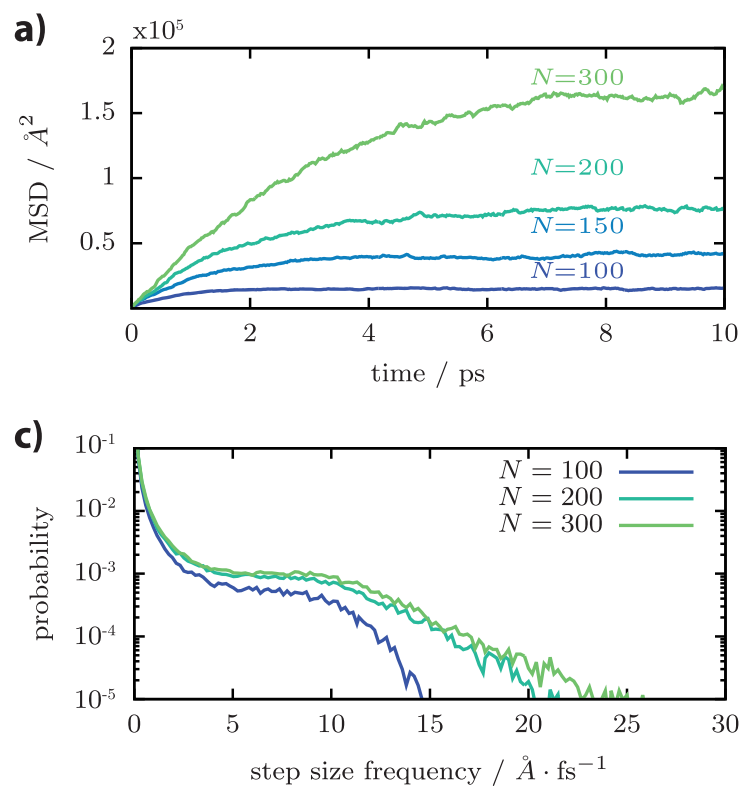

Symmetry-preserving
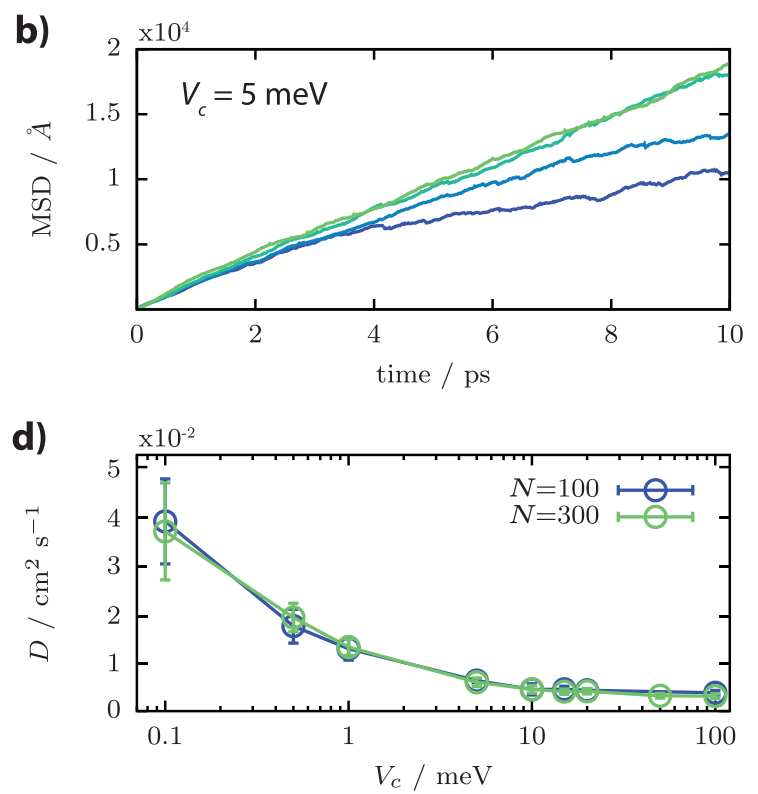

Figure 7. Comparing dynamics under symmetry-preserving and non-symmetry-preserving solutions to the TCP. (a, b) Mean-squared displacement (MSD) for excitons on polymer chains with varying number of monomer units, $N$. Panel a is computed with the non-symmetry-preserving overlap method, and panel b is computed with our symmetry-preserving dynamics. For each polymer size, data is averaged over 3000 independent trajectories carried out adiabatically in the lowest energy excited state. (c) Step size distribution for exciton displacements in the non-symmetrypreserving method showing that there are more frequent long-range hops with increasing polymer chain lengths. (d) Dependence of diffusivity, $D$, on $V_{\mathrm{c}}$ in the symmetry-preserving method. Steady state diffusivity is shown to be independent of polymer chain lengths (e.g., $N=100$ and 300$)$. Error bars denote one standard deviation uncertainty among 10 samples, each averaged over 1000 independent trajectories.

MSD data that are plotted in Figure $7 b$, we find that the diffusivity at short times $(t<500 \mathrm{fs})$ is generally faster than that at longer times. We attribute this difference to changes in the classical degrees of freedom, particularly the torsional landscape, that occur upon excitation. These changes result in self-trapping, which reduces the mobility of the exciton. Further evidence for self-trapping can be found by computing the time dependent average of the exciton IPR. As plotted in Figure 8 , we find a rapid decrease in exciton size during the first $\sim 100$ fs immediately following excitation.

At longer times (i.e., $t \gtrsim 5 \mathrm{ps}$ ), we observe a significant difference between the IPR computed with the symmetrypreserving and non-symmetry-preserving methods. Specifically, the non-symmetry-preserving method leads to excitons that are more delocalized than with the symmetry-preserving method. We observe that the IPR for unmodified purely adiabatic dynamics is essentially the same as that with the symmetrypreserving method, indicating that the symmetry-preserving method effectively populates nodeless and torsionally relaxed adiabatic states. On the other hand, the tendency for the nonsymmetry-preserving approach to populate higher energy states $(n>1)$, which are inherently more delocalized (see Figure 6), leads to a small but unintended increase in mean exciton size.

The two dominant modes of exciton mobility in long chain conjugated polymers are the diffusion of the active diabat along the length of the polymer chain, as mediated by torsional fluctuations in the tails of the active diabatic state, and hopping through avoided crossings. Since $V_{c}$ controls the threshold for this hopping mobility, it thus also contributes significantly to the determination of exciton diffusivity. This can be seen in Figure $7 d$, which contains a plot of the steady-state diffusivity

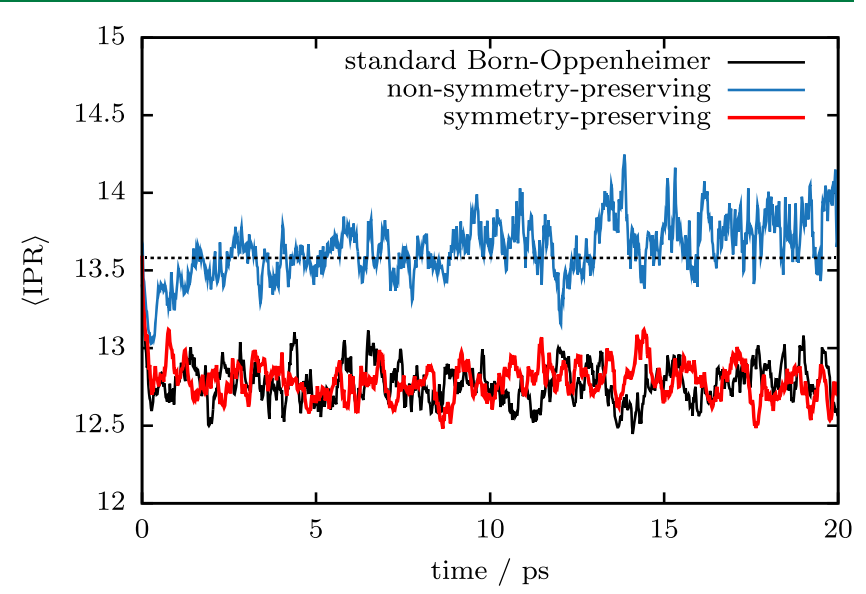

Figure 8. Comparison of the time evolution of the average inverse participation ratios (IPRs) computed on the basis of the symmetrypreserving method (red), the non-symmetry-preserving overlap method (blue), and the standard Born-Oppenheimer method (black) for 300-mer chains. The data set for the symmetry-preserving method is the same as that in Figure $7 \mathrm{~b}$. The dotted horizontal line indicates the average IPR at $t=0$, which is the same for all three methods. Each data set represents an average over 3000 independent trajectories.

as a function of $V_{\mathrm{c}}$. We find that $D$ ranges between values of 0.005 and $0.04 \mathrm{~cm}^{2} / \mathrm{s}$, depending on the choice of $V_{c}$.

Our simulated values of diffusivity are larger than the estimated value of $D \approx 0.001 \pm 0.0004 \mathrm{~cm}^{2} / \mathrm{s}$ based on fluorescence quenching experiments by Healy et al. ${ }^{41} \mathrm{We}$ attribute this difference to the lack of disorder in monomer excitation energy and in our assumption that the polymer chain is in an ideal extended linear configuration. For instance, in the 
presence of configurational disorder, diffusivity computed from our model is about $0.001 \pm 0.0002 \mathrm{~cm}^{2} / \mathrm{s}$ at $V_{\mathrm{c}}=10 \mathrm{meV}$, which is about $20 \%$ smaller than that of linear configurations, and is also in good agreement with experimental measurements by Healy et al. We have simulated disordered polythiophene backbone configurations using the coarse grained poly(3hexylthiophene) model by Schwarz et al. $^{42}$ Details on simulations with disordered polymer configurations are described in the Supporting Information.

The large difference in steady-state diffusivity between our symmetry-preserving method and the overlap-based nonsymmetry-preserving method is not entirely due to differences in exciton size. The analysis of trajectories generated on long chains indicates that, when the nodal structure of the wave function is not preserved, the dynamics features frequent longrange hops. These long-range hops, illustrated in Figure $7 c$, serve to artificially enhance exciton diffusivity. The frequency of these hops increases with increasing chain length even when the chain is much larger than the exciton size. This finding highlights that the effect is highly nonlocal and thus inconsistent with physical expectations for these dynamics.

The origins of this nonlocal hopping behavior are the relationship between the quantum force and the exciton wave function amplitude as well as the use of wave function overlap to identify trivial crossings. As the number of nodes increases, the excitonic wave function exhibits several local maxima in space, such that the planarizing forces (eq 27) also become spatially nonlocal and lower in magnitude. These events lead to frequent long-range hopping, which is in contrast to the dynamics of the self-trapped lowest exciton state due to spatially localized planarizing forces. Moreover, the overlap method itself is not as robust in identifying trivial crossings as the joint probability density approach employed in the symmetry-preserving method. With the overlap method, the signature of a trivial crossing is when $S_{k, n}>S_{k, k}$, where $k$ is the active state index and $n \neq k$. In practice, we have determined that this method is only reliable for detecting trivial crossings when the diabatic coupling is very small, i.e., when $\left|V_{a b}\right|<0.1$ $\mathrm{meV}$, corresponding to states that are separated by more than 80 monomer units (see Figure S4). ${ }^{2,10,11}$ These results thus suggest that in certain cases, such as those we have presented here, the wave function overlap method for identifying trivial crossings can be prone to false positives and thus produce unphysical dynamics.

\section{CONCLUSION AND OUTLOOK}

We have presented a theoretical framework for solving the TCP in simulations of purely adiabatic dynamics that preserve the nodal symmetry of the wave function without the need for more expensive nonadiabatic techniques. We have shown how changes in this symmetry can lead to artificial delocalization of the wave function as well as false positives in the identification of trivial crossings.

The results presented here underscore the importance of considering the nodal structure of the adiabatic wave function when implementing dynamics that includes changes in eigenstate. This includes nonadiabatic methods based on surface hopping, which rely on gathering statistics of many adiabatic trajectories. ${ }^{6,9,13,39}$ In such approaches, artificially imposing hopping between eigenstates to prevent trivial crossings may introduce uncontrolled sources of error. In surface hopping methods, TCP is usually avoided by decreasing the time step of the simulations." ${ }^{5,9}$ Our method can be applied along the purely adiabatic segments of surfacehopping trajectories (i.e., between surface hops) to allow for larger time steps.

As we have highlighted, avoiding discontinuities in wave function nodal symmetry during simulations of adiabatic dynamics is especially important in large-scale systems with many noninteracting (uncoupled) diabatic states whose energy surfaces can potentially cross. When our symmetry-preserving method is applied to simulations of exciton dynamics on long chain conjugated polymer systems, the TCP is effectively eliminated and exciton properties are free from unphysical polymer chain length dependent artifacts. Therefore, the symmetry-preserving method for solving the TCP ensures reliable simulations for experimentally relevant system sizes, as in the case of long conjugated polymer chains (>100 monomers).

\section{ASSOCIATED CONTENT}

\section{S Supporting Information}

The Supporting Information is available free of charge on the ACS Publications website at DOI: 10.1021/acs.jctc.9b00302.

Details on algorithm implementations for adiabatic dynamics with and without symmetry preserving, comparisons of trivial crossing defection methods, and polymer model parametrization (PDF)

\section{AUTHOR INFORMATION}

\section{Corresponding Author}

*E-mail: awillard@mit.edu. ORCID ${ }^{\circ}$

Elizabeth M. Y. Lee: 0000-0001-9143-3140

Adam P. Willard: 0000-0002-0934-4737

Notes

The authors declare no competing financial interest.

\section{ACKNOWLEDGMENTS}

E.M.Y.L. acknowledges helpful discussions with Amr Dodin, Dr. Troy Van Voorhis, and Dr. David R. Yarkony. The authors thank Dr. David M. Huang for sharing his LAMMPS codes to simulate his coarse grained polymer model in ref 42 . This work was supported by the Center for Excitonics, an Energy Frontier Research Center funded by the US Department of Energy, Office of Science, Office of Basic Energy Sciences, under Award DE-SC0001088 (MIT). E.M.Y.L. has been partially supported by the U.S. National Science Foundation Graduate Research Fellowship Program under Grant No. 1122374.

\section{REFERENCES}

(1) Fabiano, E.; Keal, T.; Thiel, W. Implementation of Surface Hopping Molecular Dynamics Using Semiempirical Methods. Chem. Phys. 2008, 349, 334-347.

(2) Fernandez-Alberti, S.; Roitberg, A. E.; Nelson, T.; Tretiak, S. Identification of Unavoided Crossings in Nonadiabatic Photoexcited Dynamics Involving Multiple Electronic States in Polyatomic Conjugated Molecules. J. Chem. Phys. 2012, 137, 014512.

(3) Evenhuis, C.; Martínez, T. J. A Scheme to Interpolate Potential Energy Surfaces and Derivative Coupling Vectors Without Performing a Global Diabatization. J. Chem. Phys. 2011, 135, 224110.

(4) Plasser, F.; Granucci, G.; Pittner, J.; Barbatti, M.; Persico, M.; Lischka, H. Surface Hopping Dynamics Using a Locally Diabatic Formalism: Charge Transfer in the Ethylene Dimer Cation and Excited State Dynamics in the 2-pyridone Dimer. J. Chem. Phys. 2012, 137, 22 A514. 
(5) Meek, G. A.; Levine, B. G. Evaluation of the Time-Derivative Coupling for Accurate Electronic State Transition Probabilities from Numerical Simulations. J. Phys. Chem. Lett. 2014, 5, 2351-2356.

(6) Wang, L.; Prezhdo, O. V. A Simple Solution to the Trivial Crossing Problem in Surface Hopping. J. Phys. Chem. Lett. 2014, 5, 713-719.

(7) Meek, G. A.; Levine, B. G. Accurate and Efficient Evaluation of Transition Probabilities at Unavoided Crossings in Ab Initio Multiple Spawning. Chem. Phys. 2015, 460, 117-124.

(8) Subotnik, J. E.; Jain, A.; Landry, B.; Petit, A.; Ouyang, W.; Bellonzi, N. Understanding the Surface Hopping View of Electronic Transitions and Decoherence. Annu. Rev. Phys. Chem. 2016, 67, 387417.

(9) Wang, L.; Akimov, A.; Prezhdo, O. V. Recent Progress in Surface Hopping: 2011-2015. J. Phys. Chem. Lett. 2016, 7, 2100-2112.

(10) Nelson, T.; Fernandez-Alberti, S.; Roitberg, A. E.; Tretiak, S. Artifacts Due to Trivial Unavoided Crossings in the Modeling of Photoinduced Energy Transfer Dynamics in Extended Conjugated Molecules. Chem. Phys. Lett. 2013, 590, 208-213.

(11) Tozer, O. R.; Barford, W. Intrachain Exciton Dynamics in Conjugated Polymer Chains in Solution. J. Chem. Phys. 2015, 143, 084102.

(12) Tully, J. C. Perspective: Nonadiabatic Dynamics Theory. J. Chem. Phys. 2012, 137, 22A301.

(13) Qiu, J.; Bai, X.; Wang, L. Subspace Surface Hopping with SizeIndependent Dynamics. J. Phys. Chem. Lett. 2019, 10, 637-644.

(14) Sun, Z.; Li, S.; Xie, S.; An, Z. Solution for the Trivial Crossing Problem in Surface Hopping Simulations by the Classification on Excited States. J. Phys. Chem. C 2018, 122, 8058-8064.

(15) Wang, L.; Beljonne, D. Flexible Surface Hopping Approach to Model the Crossover from Hopping to Band-like Transport in Organic Crystals. J. Phys. Chem. Lett. 2013, 4, 1888-1894.

(16) Moriconi, M. Nodes of Wavefunctions. Am. J. Phys. 2007, 75, 284-285.

(17) Van Voorhis, T.; Kowalczyk, T.; Kaduk, B.; Wang, L.-P.; Cheng, C.-L.; Wu, Q. The Diabatic Picture of Electron Transfer, Reaction Barriers and Molecular Dynamics. Annu. Rev. Phys. Chem. 2010, 61, 149-170.

(18) Nitzan, A. Chemical Dynamics in Condensed Phases, 1st ed.; Oxford University Press: New York, 2006.

(19) Bolinger, J. C.; Traub, M. C.; Brazard, J.; Adachi, T.; Barbara, P. F.; Vanden Bout, D. A. Conformation and Energy Transfer in Single Conjugated Polymers. Acc. Chem. Res. 2012, 45, 1992-2001.

(20) Menke, S. M.; Luhman, W. A.; Holmes, R. J. Tailored Exciton Diffusion in Organic Photovoltaic Cells for Enhanced Power Conversion Efficiency. Nat. Mater. 2013, 12, 152-157.

(21) Jin, X.-h.; Price, M. B.; Finnegan, J. R.; Boott, C. E.; Richter, J. M.; Rao, A.; Menke, S. M.; Friend, R. H.; Whittell, G. R.; Manners, I. Long-Range Exciton Transport in Conjugated Polymer Nanofibers Prepared by Seeded Growth. Science 2018, 360, 897-900.

(22) Rossi, G.; Chance, R. R.; Silbey, R. Conformational Disorder in Conjugated Polymers. J. Chem. Phys. 1989, 90, 7594-7601.

(23) Simine, L.; Rossky, P. J. Relating Chromophoric and Structural Disorder in Conjugated Polymers. J. Phys. Chem. Lett. 2017, 8, 17521756.

(24) Barford, W.; Boczarow, I.; Wharram, T. Ultrafast Dynamical Localization of Photoexcited States in Conformationally Disordered Poly(p-Phenylenevinylene). J. Phys. Chem. A 2011, 115, 9111-9119.

(25) Binder, R.; Wahl, J.; Römer, S.; Burghardt, I. Coherent Exciton Transport Driven by Torsional Dynamics: A Quantum Dynamical Study of Phenylene-Vinylene Type Conjugated Systems. Faraday Discuss. 2013, 163, 205-222.

(26) Binder, R.; Lauvergnat, D.; Burghardt, I. Conformational Dynamics Guides Coherent Exciton Migration in Conjugated Polymer Materials: First-Principles Quantum Dynamical Study. Phys. Rev. Lett. 2018, 120, 227401.

(27) Liang, R.; Cotton, S. J.; Binder, R.; Hegger, R.; Burghardt, I.; Miller, W. H. The Symmetrical Quasi-Classical Approach to Electronically Nonadiabatic Dynamics Applied to Ultrafast Exciton
Migration Processes in Semiconducting Polymers. J. Chem. Phys. 2018, 149, 044101.

(28) Davydov, A. The Theory of Molecular Excitons. Usp. Fiz. Nauk 1964, 82, 393-448.

(29) Tapping, P. C.; Clafton, S. N.; Schwarz, K. N.; Kee, T. W.; Huang, D. M. Molecular-Level Details of Morphology-Dependent Exciton Migration in Poly(3-hexylthiophene) Nanostructures. J. Phys. Chem. C 2015, 119, 7047-7059.

(30) Lobaugh, J.; Rossky, P. J. Computer Simulation of the Excited State Dynamics of Betaine-30 in Acetonitrile. J. Phys. Chem. A 1999, 103, 9432-9447.

(31) Warshel, A.; Karplus, M. Calculation of Ground and Excited State Potential Surfaces of Conjugated Molecules. I. Formulation and Parametrization. J. Am. Chem. Soc. 1972, 94, 5612-5625.

(32) Pariser, R.; Parr, R. G. A Semi-Empirical Theory of the Electronic Spectra and Electronic Structure of Complex Unsaturated Molecules. I. J. Chem. Phys. 1953, 21, 466-471.

(33) Pariser, R.; Parr, R. G. A Semi-Empirical Theory of the Electronic Spectra and Electronic Structure of Complex Unsaturated Molecules. II. J. Chem. Phys. 1953, 21, 767-776.

(34) Pople, J. A. Electron Interaction in Unsaturated Hydrocarbons. Trans. Faraday Soc. 1953, 49, 1375-1385.

(35) Jailaubekov, A. E.; Willard, A. P.; Tritsch, J. R.; Chan, W.-L.; Sai, N.; Gearba, R.; Kaake, L. G.; Williams, K. J.; Leung, K.; Rossky, P. J.; Zhu, X.-Y. Hot Charge-Transfer Excitons Set the Time Limit for Charge Separation at Donor/acceptor Interfaces in Organic Photovoltaics. Nat. Mater. 2013, 12, 66-73.

(36) Hu, Z.; Willard, A. P.; Ono, R. J.; Bielawski, C. W.; Rossky, P. J.; Vanden Bout, D. a. An Insight into Non-Emissive Excited States in Conjugated Polymers. Nat. Commun. 2015, 6, 8246.

(37) Vanden-Eijnden, E.; Ciccotti, G. Second-Order Integrators for Langevin Equations with Holonomic Constraints. Chem. Phys. Lett. 2006, 429, 310-316.

(38) Barford, W.; Bittner, E. R.; Ward, A. Exciton Dynamics in Disordered Poly(p-phenylenevinylene). 2. Exciton Diffusion. J. Phys. Chem. A 2012, 116, 10319-10327.

(39) Tully, J. Molecular Dynamics with Electronic Transitions. J. Chem. Phys. 1990, 93, 1061-1071.

(40) Spörkel, L.; Thiel, W. Adaptive Time Steps in Trajectory Surface Hopping Simulations. J. Chem. Phys. 2016, 144, 194108.

(41) Healy, A. T.; Boudouris, B. W.; Frisbie, C. D.; Hillmyer, M. A.; Blank, D. A. Intramolecular Exciton Diffusion in Poly(3-hexylthiophene). J. Phys. Chem. Lett. 2013, 4, 3445-3449.

(42) Schwarz, K. N.; Kee, T. W.; Huang, D. M. Coarse-grained Simulations of the Solution-phase Self-Assembly of Poly(3-hexylthiophene) Nanostructures. Nanoscale 2013, 5, 2017-2027. 